Methotrexate (MTX), in clinical practice. Other factors associated were also investigated.

Methods: An inception cohort of GCA was assembled in the out-patient clinic at Hospital Clinico San Carlos, including patients from the date of diagnosis (Jan-1991 until Sept-2013), and followed-up until Sept-2014. Main outcome: relapses defined as after an objective improvement, patient has again symptoms or signs of GCA with high ESR and the need to increase corticosteroids at least $10 \mathrm{mg}$. The independent variable was exposure to MTX over time. Covariables: Sociodemographic, clinical, and treatment. Incidence rates of relapses (IR) per 100 patient-years with their $95 \%$ confidence intervals $[\mathrm{Cl}$ were estimated using survival techniques. Time of exposure comprised the period from diagnosis until: lost of follow-up, main outcome, exposure to MTX or the end of the study. MTX influence on IR was analyzed by multivariable Cox models.

Results: 168 patients were included (675 patient-years). $80 \%$ of them were female, with a mean age of $76 \pm 7$ years. $65 \%$ of the patients were on MTX, with mean dose of $10 \mathrm{mg} /$ week. $31 \%$ of patients had relapses with an IR of 12 [9.6-14.9]. The median number of relapses was 1 [1-2], with a median lag time of 1.6 [0.6-6.3] years. In the multivariate analysis, exposure to MTX had less risk of flaring compared to those never on MTX $(p<0.05)$. Other variables included in the final model were: visual alterations, constitutional symptoms or malaise at clinical presentation of GCA.

Conclusions: The use of MTX seems to decrease the risk of recurrences. We also found other factors influencing on flares.

Disclosure of Interest: None declared

DOI: 10.1136/annrheumdis-2017-eular.6118

\section{THU0310 FREQUENCY OF RELAPSES AND TREATMENT DISCONTINUATION DURING LONG-TERM FOLLOW-UP OF PATIENTS WITH GIANT CELL ARTERITIS}

C. Tsalapaki, A. Lazarini, K. Antonatou, K. Thomas, K. Klavdianou, C. Koutsianas, D. Vassilopoulos. Joint Rheumatology Program, Clinical Immunology-Rheumatology Unit, 2nd Department of Medicine and Laboratory, National and Kapodistrian University of Athens School of Medicine, Hippokration General Hospital, Athens, Greece

Background: There are limited data regarding the long term outcomes of patients with giant cell arteritis (GCA) in the modern therapeutic era.

Objectives: To evaluate relapse, treatment discontinuation and complication rates in GCA patients during long term follow-up.

Methods: A retrospective systematic chart review of GCA patients who were followed in an Academic Rheumatology Unit between 2002 to 2016 was performed. Demographic, clinical, laboratory and treatment data were collected and analyzed.

Results: 53 GCA patients were included in the study. $62 \%(n=33)$ were women with a mean age at diagnosis of $73 \pm 8.8$ years and median duration of symptoms of 1.3 months. 41 patients $(77 \%)$ had biopsy proven GCA while in 5 patients $(9 \%)$ there was evidence of large vessel involvement. At presentation, the most common symptoms were headache $(60 \%)$, fever $(51 \%)$, scalp tenderness $(47 \%)$, jaw claudication (39\%), visual disturbances (23\%), polymyalgia rheumatica symptoms $(9 \%)$ and vision loss $(6 \%)$. Regarding laboratory data at baseline, the median ESR and CRP were $101 \mathrm{~mm} / \mathrm{h}$ and $50 \mathrm{mg} / \mathrm{dl}$ respectively while the mean hemoglobin $(\mathrm{Hb})$ and platelet $(\mathrm{PLT})$ count was $11.3 \pm 1.2 \mathrm{~g} / \mathrm{dl}$ and $381.000 \pm 134.000$, respectively. All patients were initially treated with tapering doses of pos steroids (mean start prednisolone dose: $42 \pm 12 \mathrm{mg} /$ day) while 2 patients (4\%) were given IV steroid pulses. During follow-up (3.1 \pm 2.7 years), for patients with did not adequately respond or could not tolerate steroids, non-biologic $(n=12,23 \%)$ or biologic DMARDs $(n=4,7 \%)$ were added. Relapses requiring change in immunosuppressive therapy occurred in more than half of patients $(n=28,53 \%)$; among these $67 \%$ were laboratory and $56 \%$ clinical relapses. Osteoporosis $(17 \%)$, cataracts $(7 \%)$, fractures $(4 \%)$ and avascular necrosis $(2 \%)$ developed during chronic steroid treatment. At the last follow-up visit, $39 \%(n=21)$ of patients had discontinued steroids and $31 \%(n=17)$ all treatments. Comparing the group of patients who had discontinued treatment (D/C group) to those who were unable to stop therapy (continued therapy group), there were no statistically significant differences (age, duration of symptoms at diagnosis, initial steroid dose, baseline and follow-up ESR, CRP, $\mathrm{Hb}$ and platelet values, relapses, co-administration of DMARDs, comorbidities), except from gender (females: $\mathrm{D} / \mathrm{C}$ group $=41 \%$, continued therapy group $=72 \%, p=0.03$ ). Kaplan-Meier analysis also showed that the median time to discontinuation of treatment was longer in females compared to males (log rank $p=0.018$ ).

Conclusions: In this long-term follow-up study, relapses occurred in more than half of GCA patients while only one out of three patients were able to discontinue all therapies. Among different variables, only male sex was associated with earlier treatment discontinuation.

Acknowledgements: Supported by research grants from the Special Account for Research Grants (S.A.R.G.), National and Kapodistrian University of Athens, Athens, Greece.

Disclosure of Interest: None declared

DOI: 10.1136/annrheumdis-2017-eular.6115

\section{THU0311 FECAL MICROBIOTA IN BEHÇET'S SYNDROME PATIENTS WITH MUCOCUTANOUS AND UVEITIS INVOLVEMENT}

D. Tecer ${ }^{1}$, F. Gogus ${ }^{1}$, A. Kalkancl ${ }^{2}$, M. Erdogan ${ }^{2}$, S. Coban ${ }^{3}$,

M. Hasanreisoglu ${ }^{4}$, T. Karakan ${ }^{5} .{ }^{1}$ Department of Physical Medicine and

Rehabilitation, Division of Rheumatology; ${ }^{2}$ Department of Medical Microbiology;

${ }^{3}$ School of Medicine phase 3 student; ${ }^{4}$ Department of Ophthalmology;

${ }^{5}$ Department of Gastroenterology, Gazi University, Faculty of Medicine, Ankara,

Turkey

Background: Innate immunity has a major role in the pathogenesis of Behçet's syndrome. The gut microbiota is an active component of the immune system. It plays an important role in the formation of the immune system in the early life and in the continuation of immune homeostasis through the life. Dysbiosis, imbalance in the gut microbiota, can lead to many serious metabolic and inflammatory pathologies

Objectives: We aimed to investigate the gut microbiota structure in Behçet's syndrome patients with mucocutanous and uveitis involvement only.

Methods: 6 patients with Behçet's syndrome with uveitis, 12 patients with familial Mediterranean fever (FMF) and 9 patients with Crohn's disease (CD) and 10 healthy controls were included. Patients, positive and healthy controls were excluded if they had one of the following combined diseases/situations: a) gastrointestinal surgical history (e.g. bariatric surgery, gastrectomy or colectomy), b) antibiotic or probiotics use in the last 3 months, c) specific dietary restriction, d) malignancy, e) additional autoimmune disease or inflammatory bowel disease. Total DNA was extracted from fecal samples using the QIAamp DNA stool Mini Kit following the manufacturer's instructions (Qiagen). Next generation sequencing of 16SrRNA gene was performed using lon Torrent Technology. Partial 16S rRNA ene sequences were amplified from extracted DNA using the 16S Metagenomics Kit (Life Technologies). The integrity of the PCR amplicons was analyzed by gel electrophoresis. PCR products were purified using AMPure XP DNA purification beads and Invitrogen DynaMaq magnet apparatus. DNA concentration of the amplified sequence was equalized through the Qubit System (Life Technologies). The libraries was created by using the Ion Plus Fragment Library Kit. Barcodes were also added to each sample using the Ion Express Barcode Adapters Kit. Emulsion PCR was carried out using the lon One Touch 2 machine. Sequenciing of the amplicon libraries was carried out on a 318 chip the Ion Torrent Personal Genome Machne System and Ion PGM Hi-Q kit. 16S rRNA sequences were analyzed by lon Reporter Software.

Results: In healthy subjects, fecal microbiota consisted predominatly of Bacteroidetes $(53.2 \%)$ including Bacteroides and Prevotella genuses. Firmicutes such as Bacilli, Clostridia, followed, consisting $31.2 \%$ of the bacterial community. Fecal bacterial flora of patients with Behçet's syndrome consisted of Firmicutes $(45 \%)$, Proteobacteria $(23 \%)$ such as Enterobacteriaceae and Prevotellaceae, and Bacteriodetes (10\%). FMF cases were found to be colonized by Firmicutes $(39.3 \%)$, Bacteriodetes $(32.2 \%)$ and Proteobacteria (13.6\%) predominantly. CD cases were colonized by Enterobacteriacea $(43 \%)$, and other Proteobacteria groups, followed by Bacteriodetes (15.4\%) and Firmicutes (8.8\%).

Conclusions: Fecal flora of patients with Behçet's syndrome and of positive control groups (FMF and CD) differed significantly from that of healthy controls. In a subgroup of patients with Behçet's disease with uveitis and muscucutaneaous involvement only, firmicutes species seem to be the dominant bacterial fecal flora. Disclosure of Interest: None declared

DOI: 10.1136/annrheumdis-2017-eular.6541

\section{THU0312 PERFORMANCE CHARACTERISTICS AND PREDICTORS OF TEMPORAL ARTERY ULTRASOUND AND BIOPSY FOR THE DIAGNOSIS OF GIANT CELL ARTERITIS IN A REAL WORLD POPULATION; A PROSPECTIVE COHORT STUDY}

R. Conway ${ }^{1}$, S. Di Bello ${ }^{1}$, L. O’Neill ${ }^{1}$, G.M. McCarthy ${ }^{2}$, C.C. Murphy ${ }^{3}$,

D.J. Veale ${ }^{1}$, U. Fearon ${ }^{4}$, R.P. Killeen ${ }^{5}$, E.J. Heffernan ${ }^{5}$, E.S. Molloy ${ }^{1}$.

${ }^{1}$ Rheumatology, St Vincents University Hospital; ${ }^{2}$ Rheumatology, Mater

Misericordiae University Hospital; ${ }^{3}$ Ophthalmology, Royal College of Surgeons in Ireland; ${ }^{4}$ Molecular Rheumatology, Trinity College Dublin; ${ }^{5}$ Radiology, St

Vincents University Hospital, Dublin, Ireland

Background: The diagnosis of giant cell arteritis (GCA) remains a clinical one. Temporal artery (TA) ultrasound (US) has been proposed as a new diagnostic tool in GCA.

Objectives: To assess the performance characteristics of TA US and biopsy in routine clinical practice.

Methods: All patients presenting with suspected GCA to our institutions are recruited to a prospective registry. Patients who had both a TA US and biopsy performed at the time of presentation were included in the current study. US was performed by 2 radiologists. The performance characteristics of both tests were compared to physician diagnosis at six months following presentation. Predictive factors for positive US and biopsy were explored in univariate and multivariable logistic regression analyses.

Results: 162 patients were included, 123 (76\%) with GCA. Mean (SD) duration of glucocorticoids was 6.6 days (19.4) at the time of TA US and 6.2 days (8.4) at the time of TA biopsy. US had a sensitivity of $52.8 \%(95 \% \mathrm{Cl} 43.7,61.9)$ and specificity of $71.8 \%(95 \% \mathrm{Cl} 54.9,84.5)$. There were 11 false positive US results; 5 with migraine, 2 other vasculitides, 2 local infections, 2 malignancies. Biopsy 